Doves on Decamber 26; 9 Snowy Owls on March 3; 15 Shont-eared Owls on April 22; $950-$ Horned Larks in 33 miles in the Vanscoy-Delisle-Asquith area on March 24; 10 Black-capped Chickadees on March 2 and September 9 and 41 on December 26; 67 Mountain Bluebirds on March 24 and 145 on August 29; 75+ Myrtle Warblers on May 21; 181 Western Meadowlarks in 130 miles southwest of Saskatoon on May 28; 42 Savannah Sparrows banded at Rice Lake on September 4; 12,000土 Lapland Longspurs and Snow Buntings (a few) on May 21 between Vonda and Buffer Lake, and 4,500士 Snow Buntings on April 8.

\section{Stragglers, Rarities and Other}

\section{Abnormal Birds}

This section has been restricted to the 25 species reported only once or twice in 1967. They total 83 individuals. Except as noted in parentheses, there was a single bird per date.

Black-crowned Night Heron on April 23, Ross' Goose on September 9 and 17 (6), Cinnamon Teal on June 11, Turkey Vulture on May 31, Broadwinged Hawk on April 24 and August 26 (2), Prairie Falcon on May 18, Peregrine Falcon on May 7 and 14, Virginia Rail on September 9 (2), Piping Plover on May 27 (2) and June 4, Ruddy Turnstone on May 27 (13), Knot on May 21 (5), Whiterumped Sandpiper on May $27(20+)$ and June 10, Dunlin on May 27, Buffbreasted Sandpiper on May 27 (2), Hudsonian Godwit on May 18 (5), Black-billed Cuckoo on June 11 and
July 16 (much less common than in 1966), Saw-whet Owl on February 7, Yellow-bellied Flycatcher on August 23 and 27, Western Wood Pewee on June 11, Olive-sided Flycatcher on May 26, Boreal Chickadee on February 14 and 15, Brown Creeper on April 29 and December 23, Townsend's Solitaire on April 22 and September 22-24, Nashville Warbler on September 12 (banded), Grasshopper Sparrow on June 15.

Single partial albino House Sparrows were seen on May 6, September 13 and February 1-20. Single Brewer's Blackbirds with a few pure white feathers were reported for June 12 and September 2.

\section{Habitat Changes}

Tree clearing has continued at a rapid rate. The Hudson Bay Slough was largely destroyed to make way for a divided highway. On the other hand, three permanent bodies of water were created in and adjacent to the Saskatoon district. Brightwater Reservoir is on Beaver Creek just south of the district, while Blackstrap Reservoir, east of Dundurn, and Bradwell Reservoir, south of Bradwell, are within the area.

\section{STEVE A. MANN}

As we go to press we learn of the death on August 10 of Steve Mann, Piapot. His many friends in the SNHS send heartfelt condolences to his wife and family.

\title{
EIGHTH ANNUAL MAY BIRD CENSUS, REGINA
}

Regina's annual May bird count was taken on May 11, 1968. A rather disappointing total of 131 species and 15,145 individual birds was reported. Several factors contributed to this low species count which incidentally equals the previous low, recorded on the first annual count in 1961; the earliest possible date (we count on the Saturday prior to the long weekend in May) eliminated a number of warblers and other species which do not usually arrive until after midMay; probably more significant was 
the extremely bad migratory weather in the five days previous to our count (very cool temperatures and strong N.W. winds). An indication of just how tricky it is to catch the species as they move north is this observation by the author; the strong S.E. wind which sprang up the night prior to the count brought in good numbers of Swainson's and Gray-cheeked thrush and large numbers of the later sparrows, in two specific locations; the same areas checked on the following day showed only the odd straggler remaining.

Of some concern was the almost total absence of owls; only one Great Horned Owl and no nests were found in the area, no Long or Short-eared were counted; only the Burrowing Owl numbers were up, 11 compared to three in 1967. Other species showing marked declines included the following; Yellow - bellied Sapsucker two compared to 70 in 1967; Purple Finch-four to 23 in 1967; Tree Swallow-26 to 110 in 1967; McCown's Longspurs - 13 compared to 228 in 1967. Species whose numbers were considerably up included Redhead Duck, American Coot, Marbled Godwit, Franklin's Gull, Horned Lark, Myrtle Warbler, Yellow-headed Blackbird; Chipping, Clay-colored, Harris', White-crowned, White-throated, Lincoln and Swamp sparrows and the Lapland Longspur which jumped to 5695 from 1179 in 1967.

Of special interest were two species seen in the city - one Blue Jay and one White-breasted Nuthatch; and reported from the Flying Creek valley was the Eastern Wood Pewee - the bird was not seen but was identified by its distinctive call. The Western Wood Pewee is a common transient and occasional resident, the Eastern has not previously been reported in the area.

SPECIES LIST (numbers in parentheses are those of May 13, 1967, presented for comparison):

Horned Grebe, 71 (78); Eared Grebe, 66 (121); Western Grebe, 41 (58) ; Pied-billed Grebe, 15 (8); Great Blue Heron, 2 (3); American Bittern, 2 (6); Mute Swan, 2 (2); Whistling
Swan, 34 (748); Canada Goose, 305 (245); Mallard, 332 and 10 eggs (353); Gadwall, 94 (40); Pintail, 64 (370); Green-winged Teai, 25 137); Blue-winged Teal, 147 (171); Widgeon, 175 (102); Shoveler, 70 (378); Redhead, 170 (42); Ring-necked Duck, 2 (7); Canvasback, 86 (94); Lesser Scaup, 355 (1158): Common Goldeneye, 1 (8) ; Bufflehead, 11 (6) ; Whitewinged Scoter, 1 (0): Ruddy Duck, 43 (83); Sharp-shinned Hawk, 1 (1); Red-tailed Hawk, 5 (5) ; Broad-winged Hawk, 1 (3) ; Swainson's Hawk, 6 (13); Rough-legged Hawk, 1 (7); Marsh Hawk, 33 (38); Sparrow Hawk, 2 (72); Gray Partridge, 5 (2) ; Sandhill Crane, 6 (0); Sora, 8 (14); American Coot, 460 (227): Sernipalmated Plover, 38 (17); Piping Plover, 1 (0): Killdeer, 153 (157); Black-bellied Plover, 1 (1): Spotted Sandpiper, 22 (24); Solitary Sandpiper, 25 (119); Willet, 55 (34); Greater Yellowlegs, 7 (82) ; Lesser Yellowlegs, 50 (416); Pectoral Sandpiper, 83 (830); White rumped Sandpiper, 1 (43); Baird's Sandpiper, 46 (50) ; Least Sandpiper, 19 (82) ; Dowitcher, 16 (63) ; Semipalmated Sandpiper, 9 (1): Marbled Godwit, 29 (3) ; Sanderling, 7 (8); Avocet, 38 (53); Wilson's Phalarope, 22 (41); Ring-billed Gull, 106 (109); Franklin's Guil, 238 (125) ; Common Tern, 22 (44); Black Tern, 10 (30); Rock Dove, 57 (51); Mourning Dove, 40 (49); Great Horned Owl, 1 (9); Burrowing Owl, 11 (3); Saw-whet Owl, 1 (0); Belted Kingfisher, 11 (11): Yellow-shafted Flicker, 42 (55); Yellow - bellied Sapsucker, 2 (70): Downy Woodpecker, 3 (0): Western Kingbird, 6 (1) ; Eastern Phoebe, 6 (1) ; Least Flycatcher, 3 (1); Eastern Wood Pewee, 1 (U) ; Horned Lark, 572 (338) ; Tree Swallow, 26 (110); Barn Swallow, 10 (21); Cliff Swallow, 1 (1); Purple Martin, 32 (12); Blue Jay, 1 (0); Black-billcd Magpie, 42 (61); Common Crow, 105 (157); Black-capped Chickadee, 1 (3); White-breasted Nuthatch, 1 (0); Brown Creeper, 1 (1) ; House Wren, 1 (0); Brown Thrasher, 3 (0); Robin, 169 (666); Hermit 'Thrush, 2 (9) ; Swainson's Thrush, 200 (467); Gray-cheeked Thrush, 93 (71); Eastern Bluebird, 1 (0); Mountain Bluebird, 3 (4); Rubycrowned Kinglet, 11 (4); Water Pipit, 23 (5); Bohemian Waxwing, 1 (0); Loggerhead Shrike, 17 (54) ; Starling, 32 (43); Orangecrowned Warbler, 21 (31): Yellow Warbler, 9 (6); Myrtle Warbler, 204 (90); Blackpoll Warbler, 1 (3) ; Palm Warbler, 13 (0); Northern Waterthrush, 6 (38); Yellowthroat, 1 (0); Redstart, 1 (0); House Sparrow, 669 (438): Western Meadowlark, $276 \quad$ (273); Yellow-headed Blackbird, 192 (23); Redwinged Blackbird, 698 (927) ; Baltimore Oriole, 1 (0); Rusty Blackbird, 2 (2) ; Brewer's Blackbird, 487 (573); Common Grackle, 179 Rose-breasted Grosbeak, 1 (3) ; Purple Finch, 4 (23) ; Rufous-sided Towhee, 3 (8); Savannah Sparrow, 55 (48); LeConte's Sparrow, 1 (0); Vesper Sparrow, 82 (38); Slate-colored Junco, 7 (64); Tree Sparrow, 1 (30) ; Chipping Sparrow, 144 (27) ; Clay-colored Sparrow, 160 (7) ; Harris' Sparrow, 177 (60); Whiteerowned Sparrow, 263 (233); White-throated Sparrow, 212 (194); Lincoln's Sparrow, 168 (68) : Swamp Sparrow, 11 (0) ; Song Sparrow, 21 (24) ; McCown's Longspur, 13 (228); Lapland Longspur, 5695 (1179); Chestnut-collared Longspur, 55 (16).

Total species, 131. Total individuals, 15,145. Count totals and report compiled by Al and Betty Binnie, Regina. 


\begin{tabular}{|c|c|c|c|c|c|c|c|c|c|c|c|c|c|}
\hline $\begin{array}{l}\text { UoH!lueh NeXX } 8 \cdot M \cdot r \\
\text { SIWOXON }\end{array}$ & $\begin{array}{l}\text { ลे } \\
\text { \& }\end{array}$ & & & r. & 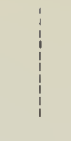 & $\stackrel{\infty}{\infty}$ & i & $\begin{array}{l}\stackrel{\infty}{N} \\
\stackrel{2}{<}\end{array}$ & & & $\begin{array}{l}\stackrel{2}{N} \\
\frac{2}{<}\end{array}$ & $\begin{array}{l}\stackrel{o}{N} \\
\stackrel{2}{\Sigma}\end{array}$ & $\begin{array}{l}\stackrel{0}{2} \\
\stackrel{2}{\Sigma}\end{array}$ \\
\hline $\begin{array}{l}\text { amo!y p!^eo } 8 \text { uets } \\
\text { NIMVdIN }\end{array}$ & $=$ & $\begin{array}{l}\stackrel{D}{N} \\
\stackrel{2}{\Sigma}\end{array}$ & $\begin{array}{l}a \\
2\end{array}$ & $\begin{array}{l}\bar{z} \\
\frac{2}{4}\end{array}$ & m & $\begin{array}{l}1 \\
\frac{2}{8}\end{array}$ & $\begin{array}{l}\text { ลิ } \\
\text { ¿ }\end{array}$ & $\frac{a}{2}$ & $\begin{array}{l}N \\
\simeq\end{array}$ & $\begin{array}{l}\stackrel{0}{N} \\
\stackrel{2}{\Sigma}\end{array}$ & $\begin{array}{l}\stackrel{L}{N} \\
\stackrel{2}{\&}\end{array}$ & $\begin{array}{l}\stackrel{o}{N} \\
\stackrel{2}{\Sigma}\end{array}$ & $\begin{array}{l}\bar{N} \\
\frac{2}{4}\end{array}$ \\
\hline $\begin{array}{l}(\cdot s \text { W) uoog } \exists \\
\exists X \forall 7 \text { ᄉXכחד }\end{array}$ & $\frac{0}{4}$ & 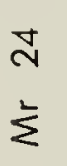 & 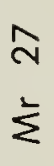 & $\begin{array}{l}\stackrel{m}{N} \\
\dot{\Sigma}\end{array}$ & $\begin{array}{l}2 \\
\frac{0}{4}\end{array}$ & $\stackrel{\infty}{\frac{\infty}{4}}$ & & $\begin{array}{l}\stackrel{0}{\Sigma} \\
\stackrel{2}{\Sigma}\end{array}$ & & & & $\begin{array}{l}\square \\
\grave{\Sigma}\end{array}$ & \\
\hline 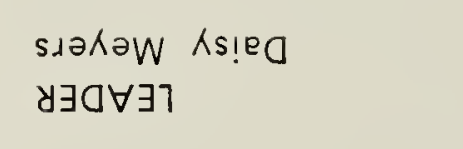 & 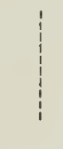 & in & 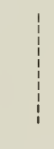 & & 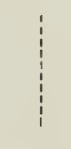 & $\begin{array}{l}\simeq \\
\frac{2}{4}\end{array}$ & 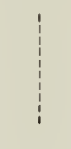 & $\begin{array}{l}\text { ָิ } \\
\stackrel{2}{<}\end{array}$ & & & & & 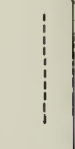 \\
\hline 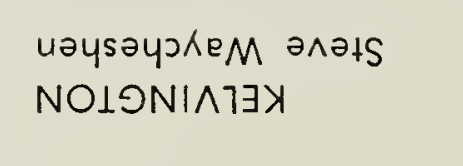 & $\stackrel{2}{i}$ & $\begin{array}{l}\text { N } \\
\Sigma\end{array}$ & $\begin{array}{l}0 \\
\frac{2}{4}\end{array}$ & $\frac{0}{4}$ & $\frac{N}{2}$ & $\frac{0}{2}$ & $\begin{array}{l}\text { ㄱ } \\
\frac{0}{4}\end{array}$ & $\begin{array}{l}\text { N } \\
\frac{0}{<}\end{array}$ & - & & $\begin{array}{l}a \\
\frac{2}{4}\end{array}$ & $\begin{array}{l}\simeq \\
\subsetneq\end{array}$ & $\begin{array}{l}a \\
\frac{2}{<}\end{array}$ \\
\hline $\begin{array}{l}\text { әueols әuUe! } \\
\text { NOISNIATEX }\end{array}$ & 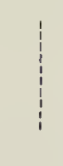 & $\begin{array}{l}\grave{N} \\
\grave{\Sigma}\end{array}$ & $\begin{array}{l}a \\
\frac{2}{8}\end{array}$ & $\frac{a}{4}$ & 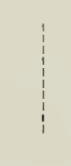 & $\begin{array}{l}= \\
\frac{2}{4}\end{array}$ & & $\begin{array}{l}\stackrel{一}{2} \\
\stackrel{2}{4}\end{array}$ & 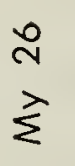 & $\begin{array}{l}\hat{N} \\
\grave{\Sigma}\end{array}$ & $\frac{a}{2}$ & \begin{tabular}{l|l}
$\sim$ \\
$\subsetneq$
\end{tabular} & $\begin{array}{l}\stackrel{n}{2} \\
\frac{2}{4}\end{array}$ \\
\hline 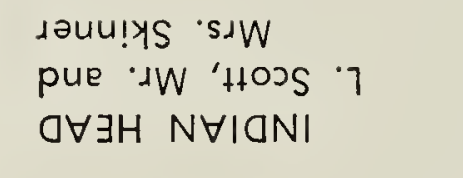 & $\begin{array}{l}\frac{0}{2} \\
\frac{2}{4}\end{array}$ & $\stackrel{\Sigma}{\Sigma}$ & $\begin{array}{l}\text { N } \\
\sum\end{array}$ & $\begin{array}{l}\stackrel{M}{N} \\
\stackrel{\Sigma}{\Sigma}\end{array}$ & $\begin{array}{l}\underset{N}{\Sigma} \\
\stackrel{2}{\Sigma}\end{array}$ & $\begin{array}{l}\bar{N} \\
\grave{\Sigma}\end{array}$ & $\begin{array}{l}\stackrel{\infty}{N} \\
\stackrel{2}{<}\end{array}$ & $\begin{array}{l}\stackrel{2}{2} \\
\stackrel{0}{4}\end{array}$ & 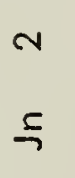 & $\begin{array}{l}\stackrel{D}{N} \\
\stackrel{2}{\Sigma}\end{array}$ & $\begin{array}{l}\circ \\
\stackrel{2}{<} \\
\stackrel{2}{<}\end{array}$ & $\begin{array}{l}\cong \\
\grave{\Sigma}\end{array}$ & $\stackrel{\infty}{\grave{\Sigma}}$ \\
\hline $\begin{array}{l}u ! \| \text { eJ } W \cdot \exists \\
N \forall S \perp y O \exists\end{array}$ & 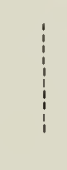 & $\begin{array}{l}\bar{m} \\
\bar{\Sigma}\end{array}$ & $\begin{array}{l}m \\
\frac{2}{4}\end{array}$ & $\begin{array}{l}m \\
\frac{m}{4}\end{array}$ & $\begin{array}{l}= \\
\frac{2}{4}\end{array}$ & $\frac{1}{2}$ & $\begin{array}{l}\hat{N} \\
\frac{2}{4}\end{array}$ & $\stackrel{2}{\frac{n}{2}}$ & m & $\begin{array}{l}\stackrel{N}{\Sigma} \\
\vec{\Sigma}\end{array}$ & $\begin{array}{l}a \\
\frac{a}{4}\end{array}$ & $\begin{array}{l}\stackrel{\mathscr{N}}{N} \\
\grave{\Sigma}\end{array}$ & $\begin{array}{l}n \\
\mathbf{z}^{2}\end{array}$ \\
\hline $\begin{array}{l}\text { HelX } \Rightarrow \cdot y \\
x S \exists\end{array}$ & $\frac{0}{2}$ & $\begin{array}{l}n \\
\frac{0}{4}\end{array}$ & $\begin{array}{l}\hat{\jmath} \\
\dot{\Sigma}\end{array}$ & $\frac{n}{\frac{0}{4}}$ & $\frac{0}{\circ}$ & $\begin{array}{l}\text { N } \\
\frac{2}{4}\end{array}$ & & $\stackrel{\stackrel{\sim}{N}}{\grave{\Sigma}}$ & & 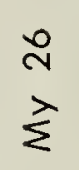 & $\begin{array}{l}\text { N } \\
\frac{2}{\alpha}\end{array}$ & $\begin{array}{l}\stackrel{o}{N} \\
\grave{\Sigma}\end{array}$ & \\
\hline 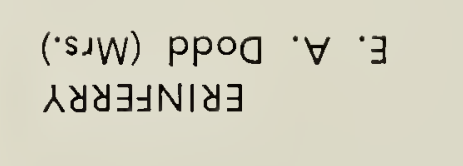 & & $\begin{array}{l}\stackrel{ }{2} \\
\stackrel{0}{<}\end{array}$ & $\begin{array}{l}\stackrel{1}{\frac{2}{4}} \\
\frac{0}{2}\end{array}$ & $\begin{array}{l}\stackrel{ }{1} \\
\frac{0}{4}\end{array}$ & 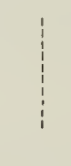 & & $\begin{array}{l}\cong \\
\stackrel{2}{\Sigma}\end{array}$ & $\frac{N}{\stackrel{N}{\Sigma}}$ & $\begin{array}{l}n \\
\dot{\Sigma}\end{array}$ & & 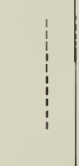 & $\begin{array}{l}N \\
\subsetneq\end{array}$ & $\begin{array}{l}\infty \\
\stackrel{\infty}{*} \\
\stackrel{2}{\alpha}\end{array}$ \\
\hline 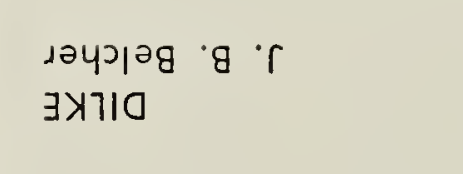 & $\begin{array}{l}\frac{0}{2} \\
\frac{0}{4}\end{array}$ & $\begin{array}{l}\stackrel{+}{2} \\
\stackrel{\Sigma}{\Sigma}\end{array}$ & 을 & $\begin{array}{l}\stackrel{D}{N} \\
\dot{\Sigma}\end{array}$ & $\begin{array}{l}\bar{m} \\
\dot{\Sigma}\end{array}$ & $\begin{array}{l}\frac{0}{2} \\
\frac{2}{4}\end{array}$ & & ${ }^{0}$ & & & $\begin{array}{c}\infty \\
\stackrel{\infty}{0} \\
\stackrel{0}{<}\end{array}$ & $\begin{array}{l}\stackrel{N}{N} \\
\grave{\Sigma}\end{array}$ & \\
\hline 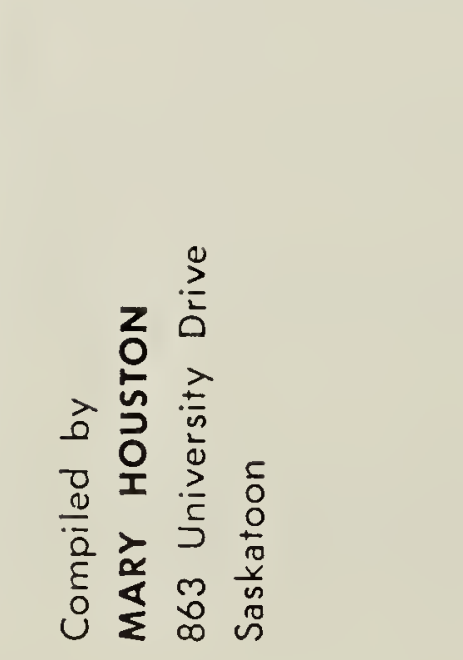 & 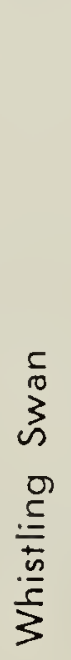 & $\begin{array}{l}0 \\
0 \\
0 \\
8 \\
0 \\
0 \\
0 \\
0 \\
0 \\
0 \\
0\end{array}$ & 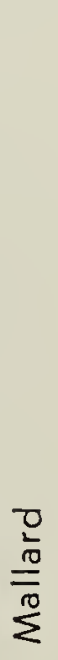 & 竞 & 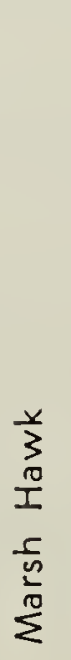 & 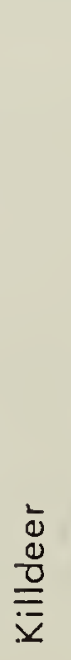 & 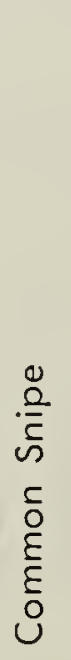 & $\begin{array}{l}0 \\
2 \\
0 \\
0 \\
0 \\
.5 \\
5 \\
5 \\
\sum^{0}\end{array}$ & 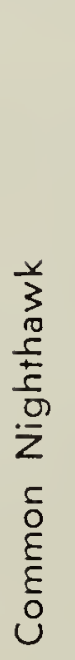 & 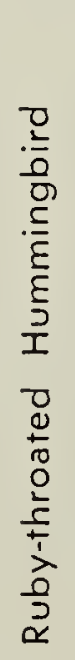 & 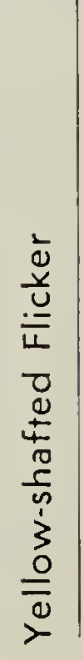 & 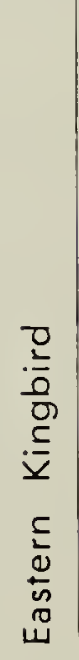 & 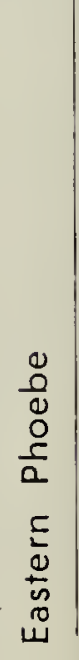 \\
\hline
\end{tabular}




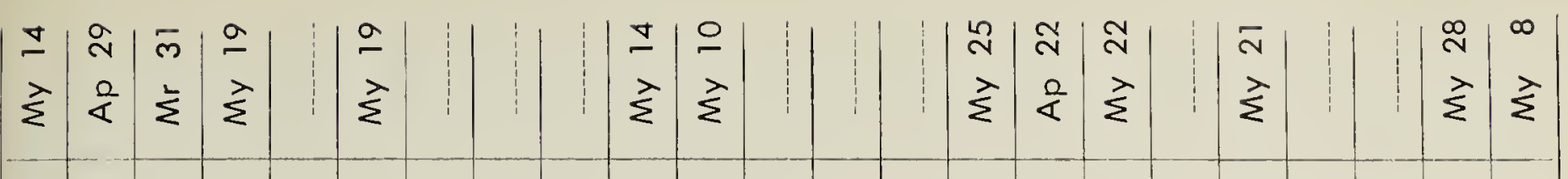

$\therefore$ ก 文与

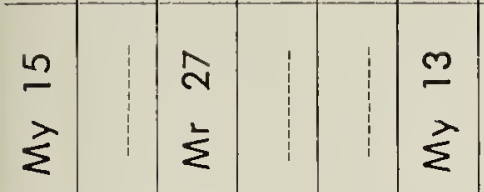
8

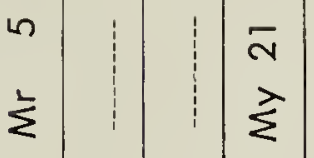
$\begin{array}{lll}\stackrel{\infty}{N} & \stackrel{\infty}{N} \\ \stackrel{2}{<} & \stackrel{2}{\sum}\end{array}$ 8

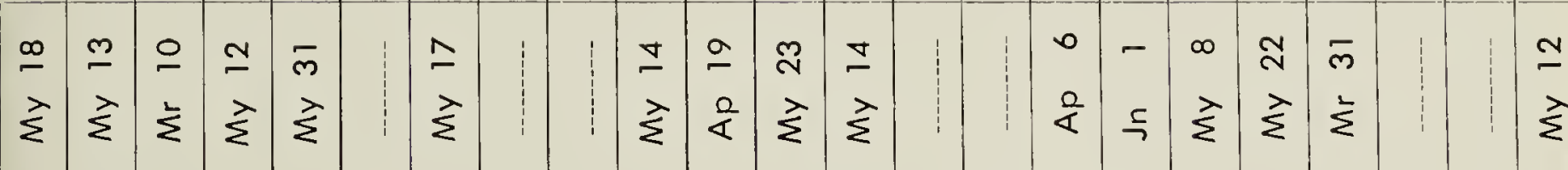

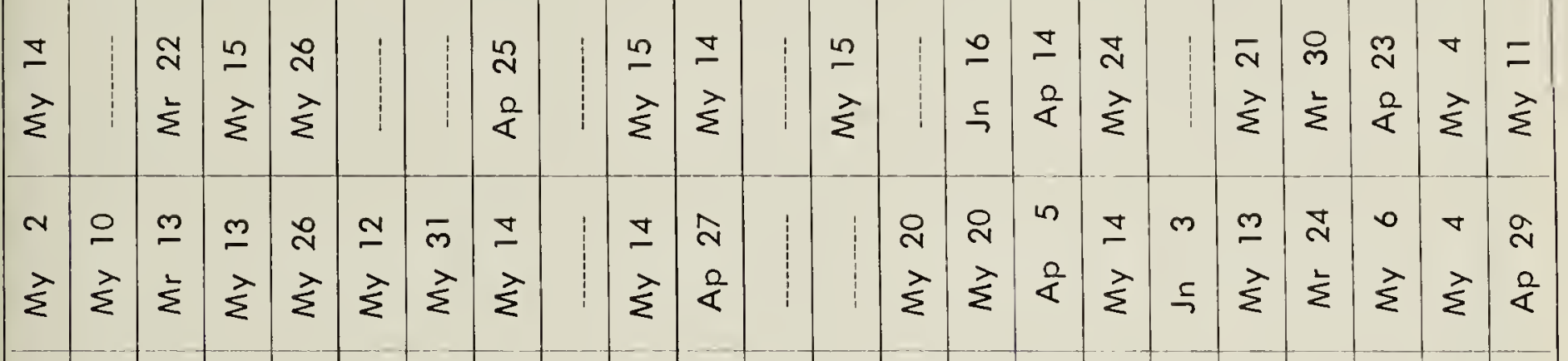

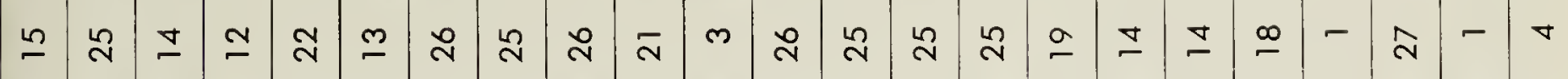

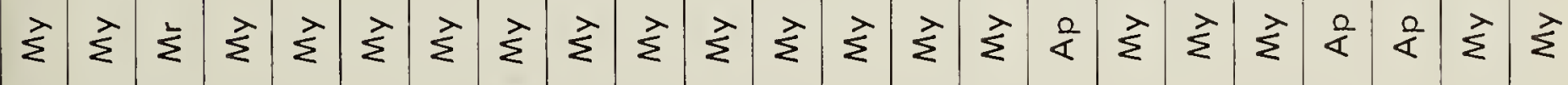

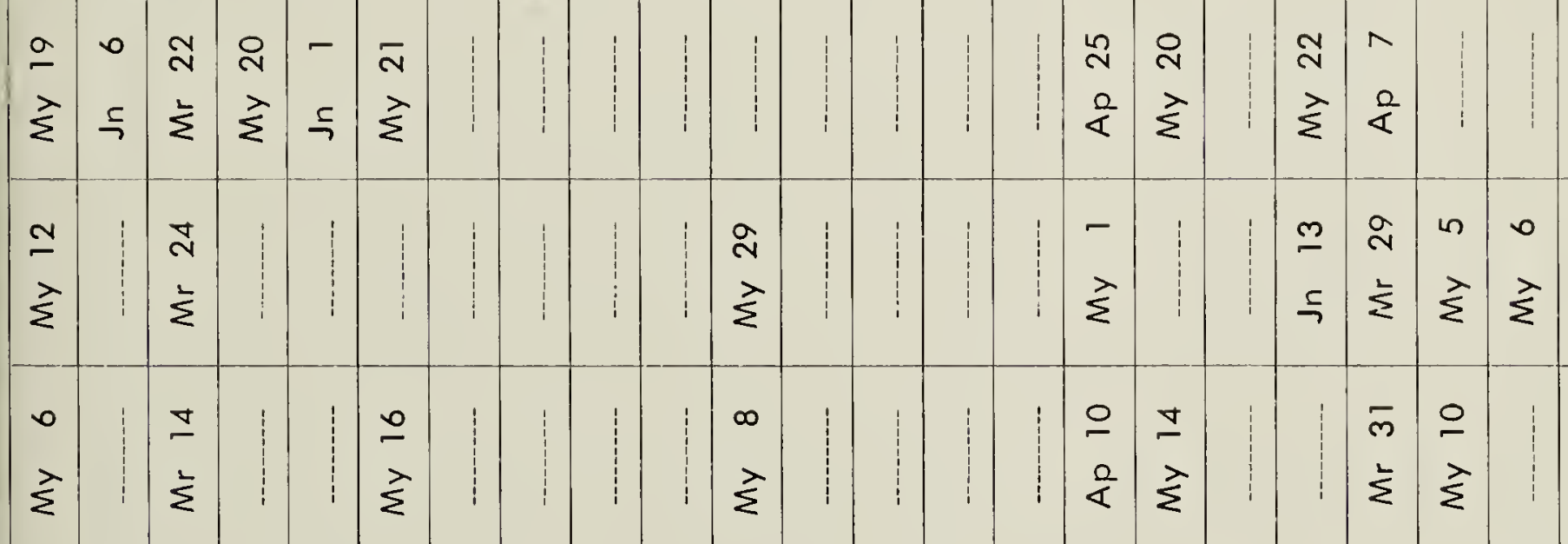

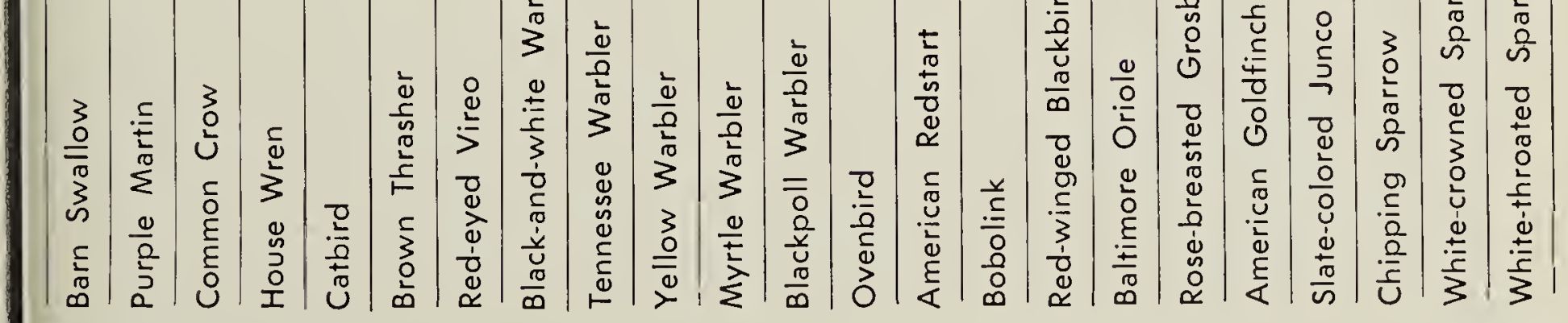




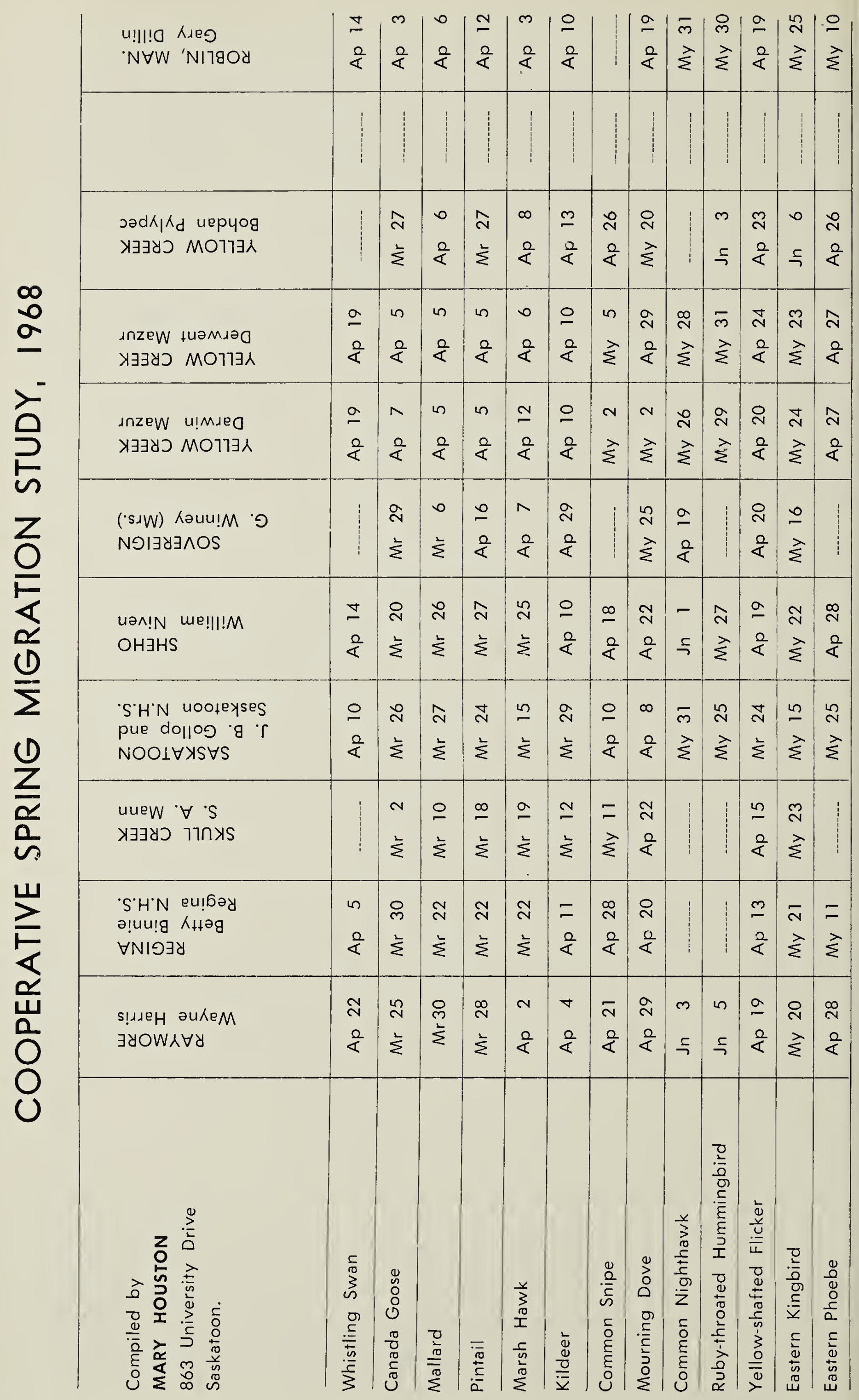


